\title{
Enfoque de Género en el acuerdo de paz entre el Gobierno Colombiano y las FARC-EP: transiciones necesarias para su implementación
}

\section{The Gender Focus in the Peace Agreement between the Colombian Government and the FARC-EP: the Transitions Needed for its Implementation}

\author{
Jakeline Vargas $^{1}$ \\ Ángela Díaz Pérez ${ }^{2}$ \\ Universidad Autónoma de Bucaramanga (Colombia)
}

Recibido: 11-10-17

Aprobado: 10-01-18

\section{Resumen}

La transversalización del Enfoque de Género en los acuerdos de paz entre el Gobierno de Colombia y la guerrilla de las FARC-EP ha sido reconocida internacionalmente como un hito que puede contribuir a transformar, a partir de éste, los procesos de paz venideros alrededor del mundo. Esta incorporación no habría sido posible sin la lucha constante de los movimientos de mujeres desplazadas, campesinas, feministas, entre otros, que siempre han trabajado activamente en la construcción de paz, mucho antes del inicio de las negociaciones. Las mujeres colombianas ya construían paz en medio del conflicto. A pesar de la importancia de sus logros, el camino no fue fácil y la incorporación de la perspectiva de género provocó intensos debates en los que se refleja que las transiciones necesarias para la sostenibilidad de la paz, más allá de la tregua entre las partes negociadoras, está en el cambio de imaginarios que la sociedad civil ha de realizar para superar los arraigados patrones culturales y valores discriminatorios que impiden el avance de la sociedad colombiana.

\footnotetext{
${ }^{1}$ (jvargas557@unab.edu.co). Máster en Políticas Públicas y Gerenciamiento del Desarrollo de la Universidad Nacional de San Martín. Docente Investigadora de la Facultad de Ciencias Jurídicas y Políticas de la Universidad Autónoma de Bucaramanga, Colombia.

2(adiaz789@unab.edu.co). Doctora en Género, Conocimiento, Subjetividad y Cultura de la Universidad de Valencia y Docente Investigadora de la Facultad de Ciencias Jurídicas y Políticas de la Universidad Autónoma de Bucaramanga, Colombia.
} 
Palabras-clave: Enfoque de género, Acuerdos de Paz, conflicto armado colombiano.

\begin{abstract}
The gender focus contained in the Peace Agreement between the Colombian Government and the guerilla group FARC-EP has been internationally recognized as a watershed moment that could contribute to future peace processes around the world. This inclusion would not have been possible without the constants struggles of the women's movements representing displaced people, peasants, and feminists, among others. Long before the negotiations even started, Colombian women had been actively working to build peace in the midst of the conflict. Despite the importance of their achievements, the road has not been easy. To include a gender perspective stimulated intense debates revealing that peace goes far beyond a truce between negotiating parties. The transitions needed for a sustainable peace requires changes in the imagination of the civil society to overcome the rooted cultural and discriminatory patterns that prevent Colombian society to move forward.
\end{abstract}

Key-words: Gender Perspective, Peace Agreement, Colombian Armed Conflict.

\title{
1. Introducción
}

La adopción de una perspectiva de género ha sido desde el año 2000 una solicitud que el Consejo de Seguridad de las Naciones Unidas a través de la Resolución 1325, les ha hecho a todos los actores que participan en la negociación y aplicación de acuerdos de paz (Numeral 8)³. La Resolución, exhorta a que se reconozcan las afectaciones diferenciales que los conflictos armados producen en la vida de las mujeres, sin desestimar su capacidad de agencia para la construcción de paz.

En ese sentido, la agenda de mujer, paz y seguridad instaurada por la $\mathrm{R} / 1325$ ha sido nutrida con la adopción de siete resoluciones más, que como señala UN WOMEN (2015:44): "exigen no solo la plena participación e

\footnotetext{
${ }^{3}$ Numeral 8 de la RES/1325(2000): "Pide a todos los que participen en la negociación y aplicación de acuerdos de paz que adopten una perspectiva de género, en que se tengan en cuenta y se incluyan, entre otras cosas: a) Las necesidades especiales de las mujeres y las niñas durante la repatriación y el reasentamiento, así como para la rehabilitación, la reintegración y la reconstrucción después de los conflictos; b) Medidas para apoyar las iniciativas de paz de las mujeres locales y los procesos autóctonos de solución de conflictos y para hacer participar a las mujeres en todos los mecanismos de aplicación de los acuerdos de paz; c) Medidas que garanticen la protección y el respeto de los derechos humanos de las mujeres y las niñas, particularmente en lo relativo a la constitución, el sistema electoral, la policía y el sistema judicial" (2000: 3).
} 
inclusión de las mujeres en todos los niveles de adopción de decisiones, sino también la protección y promoción de los derechos de la mujer en la prevención de conflictos".

No obstante, el balance de la R/1325 a quince años de su implementación, señala la persistencia de la infrarrepresentación de las mujeres en los procesos de paz y transición. Esto tiene que ver con factores relacionados a los mismos procesos y con un conjunto de factores contextuales que Paffenholz, Ross, Dixon, Schluchter \& True (2016:10) señalan a continuación:

(...) apoyo o resistencia de las élites; opinión pública; influencia de actores regionales e internacionales en el proceso de paz; presencia de grupos sólidos de mujeres; preparación de las mujeres; heterogeneidad de identidades femeninas; actitudes y expectativas sociales y políticas sobre los roles de género; redes regionales e internacionales de mujeres y existencia de compromisos previos de sensibilidad sobre cuestiones de género e inclusión de la mujer.

La interacción conjunta de los anteriores factores puede generar un ambiente más o menos favorable para que las mujeres puedan particicipar y ejercer influencia en la incorporación de sus reivindicaciones en los textos de los acuerdos. Al respecto, UN WOMEN (2015:44) aporta evidencia indicando que de los 1168 acuerdos de paz firmados entre enero de 1990 y enero de 2014, solo un $18 \%$ hace alguna referencia a las mujeres o al género. Señala también que antes de la aprobación de la Resolución 1325, solo el 11\% hacía alguna mención y después del 2000, este porcentaje aumentó al 27\%.

El balance de implementación de la R/1325 muestra una baja incorporación de la perspectiva de género en los acuerdos de paz en el mundo y la invisibilización de las mujeres como participantes que pueden influenciar los procesos de negociación política, su implementación y la sostenibilidad de lo pactado.

La correlación positiva entre la influencia ejercida por las mujeres y la firma e implementación de acuerdos fue establecida por Paffenholz et al. (2016: 6), a partir del estudio de 40 casos de negociaciones de paz. El equipo investigador logró constatar una mayor probabilidad de que se firmasen acuerdos finales en los contextos en los que ciertos grupos de mujeres pudieron ejercer una fuerte influencia en los procesos de negociación, que aquellos casos en los en los que la influencia de las mujeres era moderada, débil o inexistente, en la práctica.

En el caso colombiano, el movimiento social de mujeres participó activamente en el proceso de negociación entre el Gobierno y las Fuerzas Armadas Revolucionarias de Colombia - Ejército del Pueblo - FARC-EP, desplegando un repertorio amplio de modalidades de inclusión que logró influir significativamente a distintos actores en todas las fases del proceso de paz en Colombia. 
Es por eso que el presente artículo plantea, en primer lugar, la necesidad de incorporar el género como una categoría transversal, tanto en el estudio retrospectivo del conflicto armado como en la etapa de implementación de lo pactado tras el Acuerdo de paz. Posteriormente, usando la tipología de evaluación de la inclusión de las mujeres en los procesos de paz elaborada por Paffenholz et al. (2016: 6), se realiza una aproximación al camino recorrido para la incorporación del enfoque de género en el Acuerdo de Paz. Por último, se abordan las implicaciones de hacer una transición, de una sociedad en conflicto por más de medio siglo, a una que aporte individual y colectivamente a la construcción de una paz estable y duradera.

\section{Marco teórico}

El género, como categoría de análisis, ha ido complejizándose a partir de elementos aportados desde diferentes perspectivas teóricas, consolidándose en el tiempo como un enfoque teórico-metodológico con capacidad de abordar de forma transversal una gran diversidad de dinámicas sociales. Entre ellas, la que aquí nos ocupa, es decir, cómo esta perspectiva contribuye a la comprensión de contextos de conflicto armado y cuál es su influencia en la construcción de una paz sostenible (Mendia, 2010).

Algunos elementos clave dentro de dicha evolución, están, por ejemplo, en la diferenciación entre los conceptos de sexo y género que supera una visión centrada en las diferencias anatómicas de mujeres y hombres (Stolcke, 2000; Bonilla, 2010). También, la evolución desde los estudios sobre mujeres hacia otros que permitan entender las relaciones de poder establecidas culturalmente y que afectan al conjunto de la sociedad (Scott, 1990); o las contribuciones de la teoría crítica feminista, que han permitido afianzar y dar contenido al concepto de género. Igualmente, ha de destacarse la importancia de su abordaje teniendo en cuenta el contexto específico en el que se sitúa (Harding, 1986; Haraway, 1989, 1995) y puede afirmarse que cuenta con un carácter dinámico, en constante construcción en relación a diferentes momentos históricos y contextos socioculturales. Por esta razón, su significado no es único y puede variar.

El concepto de género hace referencia a un conjunto de prácticas, asignaciones, ideas y representaciones que culturalmente se atribuye a cada sexo como mandato social, estableciendo lo que cada uno de ellos debería ser (Scott, 1996; Lamas, 2000, 2007; Murguialday, 2000). Es decir, pone de manifiesto la diferencia entre las características biológicas predeterminadas, y aquellas construcciones culturales que han sido naturalizadas, contribuyendo a marcar las desiguales relaciones de poder entre mujeres y hombres. 
A partir del reconocimiento de esas diferencias de género, se desarrolla el enfoque analítico o perspectiva de género, la cual puede definirse en tres dimensiones: como una forma de observar y pensar los procesos, las necesidades y las demandas sociales; como un marco teórico para entender las desigualdades de género, y finalmente, como una herramienta de análisis que incorpora de manera sistemática el principio de igualdad de oportunidades entre mujeres y hombres (Likadi, 2004).

El potencial transformador de esta perspectiva es además una apuesta política para la construcción de paz, pues no puede hablarse de la existencia de conflictos armados que sean neutros al género, en tanto que estos se desarrollan en contextos socio-culturales determinados. Cuando en dichos contextos las relaciones de poder entre hombres y mujeres están insertas en el sistema de dominación patriarcal, como ocurre en el caso colombiano, las violencias ejercidas contra las mujeres en el ámbito privado llegan a ser normalizadas culturalmente ${ }^{4}$.

Si ese contexto cotidiano es repensado a la luz de las consecuencias de un conflicto armado, se evidencia que las violencias ejercidas en contra de las mujeres en dichos contextos son una exacerbación de esa cotidianidad violenta, potenciando y reproduciendo las desigualdades y la dominación de los hombres sobre las mujeres en todos los ámbitos. Como consecuencias de esta realidad, los impactos sobre las vidas y los cuerpos de las mujeres y los hombres, en los contextos de conflicto, son siempre diferenciados (Meertens, 2000, 2012). Como lo expresa Londoño (2006), desde una perspectiva feminista:

"la explicación de la violencia contra las mujeres después de un conflicto armado se enmarca en el continuum de violencia -política, económica y socialque es socialmente invisible durante la "paz", debido sobre todo a la aceptación cultural de una jerarquía de género basada en la supremacía de los hombres y la subordinación de las mujeres. Como consecuencia, en diferentes situaciones de posguerra, la violencia contra las mujeres en los hogares y otros tipos de abusos son con frecuencia considerados como un problema menor, "de mujeres", poco relevante en relación a otros" (2006:31)

En Colombia, esa reproducción de las opresiones cotidianas, exacerbadas por el conflicto, se ha manifestado especialmente, en la utilización de la violencia sexual ejercida principalmente sobre las mujeres, niñas y niños ${ }^{5}$, y aún invisibilizada, aquella sufrida por personas de género no heteronormativo,

\footnotetext{
${ }^{4}$ Reflejo de esta realidad, son las cifras reportadas por el Instituto Nacional de Medicina Legal y Ciencias Forenses en los datos del Boletín Epidemiológico: Violencia de Género. En el que se refleja que, a pesar de la jurisprudencia existente en torno a la violencia de género, Colombia pasó de tener 44.228 casos en 2014 a 49.712 en 2016. (2016:11)

${ }^{5}$ Información detallada al respecto se encuentra en el informe sobre la Resolución 1280 de 2008 del Consejo de Seguridad de la Organización de las Naciones Unidas - ONU.
} 
en especial mujeres transexuales. Como lo manifiesta Díaz (2016): "El sistema patriarcal, es la causa de esa jerarquización de valores que ha permitido que la violencia sexual afecte de forma directa y mayoritaria, a las mujeres como mecanismo para destruirlas, para intentar borrar su identidad y para dominar al enemigo" (2016:152).

Otras manifestaciones comunes son la esclavización de las mujeres para las labores domésticas o para el ejercicio de la prostitución por parte de los grupos armados, todas estas formas de control establecidas en zonas de guerra, claramente atravesadas por las desigualdades en las relaciones de poder entre hombres y mujeres que establece el sistema patriarcal (Murguialday, 2000).

A partir de dichas diferenciaciones, toma mayor relevancia la incorporación en los acuerdos de paz del enfoque diferencial, y dentro de las diferentes vertientes del mismo, el enfoque diferencial de género. Siguiendo a Arteaga (2012), quien nos habla de éste como una herramienta para la construcción de paz fundamentada en la triada justicia, equidad e igualdad, este enfoque "nace -en principio- con la pretensión de ofrecer un panorama de restitución de derechos a víctimas del conflicto desde sus particularidades; parte, por tanto, de la noción de diversidad y vulnerabilidad de estas personas, por lo cual, su interés se centra en "devolver derechos" de manera efectiva" (2012:15).

Como lo expresa la autora, el enfoque diferencial ha jugado un papel fundamental en la exigibilidad de derechos de las víctimas pertenecientes a diversos grupos sociales, consiguiendo que las respuestas estatales tengan en cuenta "la diversidad étnica, cultural, de edad, de género y de condición física y psicológica de las poblaciones objetivo a las cuales se dirigen" (2012:16); siendo reconocida esta necesidad por el Congreso de la República en el artículo 13 de la Ley 1448 de $2011^{6}$.

Además del enfoque de género y del enfoque diferencial, el análisis de los procesos de desarme, desmovilización y reintegración (DDR), requiere comprender desde una mirada integral los diferentes aspectos que inciden en la vida de las personas afectadas por entornos de conflicto y posconflicto, ya sea como víctimas o como victimarias. El concepto de interseccionalidad permite entender los diversos tipos de opresiones y relaciones de poder que se entrecruzan en la vida de una persona de acuerdo con su contexto, y en relación con elementos como la clase social, la etnia, la diversidad funcional o el género. Autoras como Hill Collins (2000) o Hancock (2007), entre otras, han reflexionado más allá del concepto, transformándolo en un paradigma

\footnotetext{
${ }^{6}$ En la Ley 1448 de 2011 del Congreso de la República, su artículo 13 manifiesta que: "El principio de enfoque diferencial reconoce que hay poblaciones con características particulares en razón de su edad, género, orientación sexual y situación de discapacidad. Por tal razón, las medidas de ayuda humanitaria, atención, asistencia y reparación integral que se establecen en la presente Ley, contarán con dicho enfoque...".
} 
teórico-metodológico dentro de la teoría critica feminista, que busca superar las limitantes dicotomías de la epistemología occidental.

Los elementos antes mencionados, enfoque de género, enfoque diferencial e interseccionalidad cumplen una importante función de complementariedad, y permiten comprender con una mayor claridad por qué la incorporación del género como eje transversal en las agendas de la paz es útil, tanto para la visibilización de los efectos negativos del conflicto, como para identificar aquellos elementos clave que contribuyen a transformar los imaginarios sociales que asignan roles fijos a las mujeres y los hombres en los contextos armados.

En este sentido, un ejemplo claro de la contribución hecha con la incorporación de estos enfoques está en transformar los imaginarios en relación con el papel que juegan las mujeres en escenarios de conflicto armado, a causa de su imagen y rol de "principales cuidadoras que se les atribuye en muchas culturas, llevan el peso de la reintegración social y psicológica de los excombatientes, especialmente aquellos que se encuentren heridos, traumatizados, enfermos" (HEGOA. 2012:19). Situación que no ha sido tenida en cuenta en muchos procesos de DDR, permitiendo resaltar el potencial y el papel estratégico que las mujeres tienen y han tenido siempre como constructoras de paz, tanto en tiempos de guerra como en etapas de implementación de acuerdos y posconflicto, e igualmente, en su relación con los grupos armados no solo como víctimas, también como victimarias.

En el caso colombiano la incorporación transversal del enfoque de género en los acuerdos, ha permitido visibilizar que trabajar desde esta perspectiva no se limita a trabajar con mujeres ${ }^{7}$, por ejemplo, identificar la importancia que supone profundizar en cuestiones como la construcción de nuevas masculinidades, encaminadas a transformar aquellas desarrolladas en los contextos de guerra, y cómo las mujeres pueden contribuir a perpetuar o no este tipo de masculinidades (Rojas y Caro, 2002).

Durante décadas el foco de atención de la investigación en relación al género y el conflicto armado en Colombia se centró en conocer los efectos perversos de la guerra sobre las mujeres. Con los procesos de paz y el inicio del DDR se han abierto nuevos retos para la investigación, de manera tal que autoras como Rojas y Caro (2002) han propuesto ejes temáticos como: postconflicto, reintegración socio-económica, justicia y Derechos Humanos, reconversión institucional e impactos del conflicto sobre las mujeres y las relaciones de género, (2002:51-52). La participación activa durante el proceso de paz y el reconocimiento como víctimas de la población LGBT ha permitido, por

7 Esta es una idea reduccionista pero frecuente en relación con el enfoque de género, se refleja incluso en muchas acciones institucionales que consideran que trabajar con grupos de mujeres es sinónimo de incorporar este enfoque. Realizar un trabajo transversal implica diferenciar el papel y las necesidades de mujeres, hombres y géneros diversos.

Araucaria. Revista Iberoamericana de Filosofia, Política y Humanidades, año 20, n 39. Primer semestre de 2018 Pp. 389-414. ISSN 1575-6823 e-ISSN 2340-2199 doi: 10.12795/araucaria.2018.i39.19 
ejemplo, abordar una realidad invisibilizada a causa de la heteronormatividad que ha regido siempre los poderes políticos en Colombia. Esta trasformación también abre un nuevo campo de estudio tanto en relación a los efectos de la violencia sobre el colectivo como en la necesidad de reconocer que hacen parte no solo de las víctimas, sino de victimarios/as y que sus procesos de DDR han de tener en cuenta su orientación de género.

Igualmente, el reconocimiento de que las mujeres han sido ampliamente excluidas en los DDR, ya que en estos "lo masculino es el modelo y la unidad de medida" (Mendia, 2009:7), pone de manifiesto la necesidad de repensar los modelos de reincorporación de personas desmovilizadas desde el enfoque de género, y sus relaciones con el resto de la comunidad.

\section{El camino recorrido para la incorporación del enfoque de género en el Acuerdo final para la terminación del conflicto y la construcción de una paz estable y duradera}

En la vivencia que las mujeres colombianas han tenido del conflicto armado en sus distintas expresiones de violencia, se ha dado una perpetuación de los roles de género, consolidando una imagen colectiva de las mujeres como víctimas pasivas en la recepción de las violaciones a sus derechos e invisibilizándolas, tanto en la reivindicación de éstos, como en los procesos de construcción de la paz y mantenimiento del tejido social.

Por tanto, esa configuración de las mujeres colombianas únicamente como víctimas es una contradicción. Efectivamente lo son, pero realizando una mirada retrospectiva, se observa que han sido ellas quienes han mantenido vivo el (des) tejido social colombiano durante el conflicto y quiénes han generado el mayor número de estrategias y alternativas para la construcción de la paz en medio del conflicto. Como lo describe Cinthya Cockburn (2007:47): "En muchas regiones del país, los líderes masculinos de los derechos humanos y las organizaciones por la paz fueron asesinados o desaparecieron". Por tanto, son las organizaciones de mujeres las que han permitido dar continuidad en la búsqueda digna de una democracia real con justicia social, que garantice una paz sostenible.

Las mujeres dejaron de ser víctimas y devinieron sujetas de derecho, crearon mecanismos de resistencia y formas de construir paz, acordes a sus múltiples identidades, confrontando la violencia con estrategias colectivas y noviolentas. Según el informe Voces de Mujeres, realizado por la Ruta Pacífica de las Mujeres $^{8}$, se refleja cómo un $66 \%$ de las mujeres que prestaron su testimonio

\footnotetext{
${ }^{8}$ La Ruta Pacífica de las Mujeres por la Negociación Política de los Conflictos, nace a mediados de los años noventa y agrupa a más de 300 organizaciones diversas de mujeres indígenas, afrocolombianas, desplazadas, jóvenes, campesinas, pobres de zonas urbanas, entre otras, y que se definen a sí mismas como: "Un proyecto político feminista, de carácter nacional, que trabaja para 
en el informe se vieron obligadas a transformar sus roles tradicionales dentro de los nuevos papeles asumidos. Así, un $40 \%$ de ellas vio en las organizaciones de mujeres su principal herramienta de resistencia, y más de un $70 \%$ de ellas encontraron diversas estrategias organizativas para la defensa de sus derechos (2013:85).

Se han movilizado por su supervivencia y las de sus comunidades, han asumido múltiples roles (productivo, reproductivo y comunitario), desde la subjetividad, y a través de la expresión de sus sentimientos y emociones enfrentan la violencia y los retos que supone la transición de la sociedad colombiana en su nuevo escenario de implementación de los acuerdos de paz con las FARC-EP. Como lo expresa una joven activista del suroccidente colombiano:

"El trabajo que hacemos las mujeres en Colombia es fundamental, reconstruimos
el tejido social a través del trabajo comunitario, somos guardianas de la memoria
de nuestro pueblo y levantamos nuestra voz en contra de la guerra. Pero es un
trabajo muy complicado porque tenemos una cultura patriarcal muy arraigada,
donde se menosprecia e invisibiliza la labor de la mujer. Tenemos la sensación de
tener que estar siempre discutiendo para que se respete nuestro trabajo. ¿Por qué
hemos de tener al lado un hombre para que nos defienda?, o ¿por qué debemos
vivir escondiéndonos para protegernos?" Brenda (Díaz 2015:153-154)

De este rol comunitario se deriva la reivindicación por espacios públicos que puedan ser gestionados por las mujeres, permitiendo la inclusión de una parte de la historia que suele quedar invisibilizada. Las organizaciones de mujeres ${ }^{9}$ han generado pensamientos autónomos para la construcción de paz, rescatando formas tradicionales de organización comunitaria adaptadas al contexto actual, demostrando su capacidad y persistencia frente a los compromisos que han adquirido con la población. Son, ante todo, pacifistas que proponen métodos y acciones alternativas de transformación.

Las mujeres en Colombia resisten a la guerra desde la vía pacífica, alejadas de las armas y desde su identidad de género, transformando de forma simbólica diferentes elementos de su cotidianidad ${ }^{10}$ como referentes en la resistencia.

conseguir un fin negociado del conflicto armado de Colombia y hacer visibles los efectos de la guerra en la vida de sus mujeres. Nos declaramos pacifistas, antimilitaristas y constructoras de una ética de la no violencia en la que los principios fundamentales son la justicia, la paz, la igualdad, la autonomía, la libertad y el reconocimiento del otro" Tomado de: www.rutapacifica.org.co

${ }^{9}$ En los siguiente Links, puede encontrarse información acerca de diversas iniciativas de las mujeres colombianas por la paz: http://www.mujeresporlapaz.org, http://www.radiofeminista.net/colombia indice.htm, http://www.asambleaporlapaz.com, http://www.humanas.org.co/alfa/index.php, https:// www.rutapacifica.org.co/, http://organizacionfemeninapopular.blogspot.com.co/p/inicio.html.

${ }^{10}$ Como ejemplo encontramos la utilización del recurso tradicional del tejido, tan importante como medio de expresión para muchas organizaciones de mujeres. El tejido representa las conexiones con el mundo, con las personas e igualmente, el destejer, desenredar y tejer de nuevo, simboliza el ciclo creativo de la vida, la muerte, la deconstrucción y reconstrucción de los significados. Para algunas comunidades el tejido plasma su historia, los caminos que recorren, sus territorios, en resumen, toda su cosmovisión. 
Realizan estratégicamente "la deconstrucción del simbolismo dominante de violencia y guerra y su sustitución por un nuevo lenguaje visual y textual, y por rituales creativos y otras prácticas que recuperan lo que las mujeres trajeron al mundo (...) se trata de indagar y recuperar el conocimiento femenino que ha sido subordinado, silenciado y enterrado" (Cockburn 2007:233-234).

En los contextos de conflicto en el mundo, los movimientos de mujeres han estado siempre al margen de los Estados haciendo un trabajo constante en la construcción de paz $^{11}$, y Colombia no ha sido la excepción. Ello, dado que como señala HUMANAS \& CIASE (2016:5), desde mucho antes que el proceso de paz iniciara, el movimiento social de mujeres con todas sus variedades y vertientes ya venía impulsando desde los territorios, la negociación política como el camino más adecuado para terminar las guerras patriarcales.

Por eso cuando inician los diálogos en La Habana, el movimiento social de mujeres en el marco del encuentro nacional de Mujeres por la Paz, lanza un manifiesto con la consigna "La paz sin mujeres no va"12 y hace un llamado a la comunidad internacional para que apoye las iniciativas de las mujeres y sus organizaciones para contribuir al reconocimiento como interlocutoras políticas indispensables en el proceso de diálogo y de construcción de la paz.

Esta iniciativa surtió efecto, y así lo reconocen las mujeres que participaron en la mesa de diálogos en La Habana:

\begin{abstract}
"Si no hubiese habido esa fuerza de la sociedad civil y de las organizaciones sociales y de mujeres que llevan trabajando tantísimos años en este tema, difícilmente lo hubiésemos podido haber incluido. La Resolución 1325, la CEDAW, los mandatos de seguridad que emanan de la 1325, nos dan un campo muy robusto para poder movilizar a la comunidad internacional en Colombia. [...] las agencias de las Naciones Unidas sabían que era necesario que las mujeres fueran parte del proceso [...] creo que logramos contribuir como mujeres, ayudar a articular a la cooperación internacional como un catalizador de este cambio". (Entrevista 15) (HUMANAS \& CIASE 2016:27).
\end{abstract}

El movimiento de mujeres utilizó de este modo un repertorio amplio de modalidades de inclusión que logró influir significativamente a distintos actores en todas las fases del proceso de paz en Colombia. Dicho repertorio es examinado usando la tipología de Paffenholz et al. (2016:6) a continuación para evaluar la inclusión de las mujeres en los procesos de paz.

\footnotetext{
${ }^{11}$ Mujeres de Negro, en Israel y Palestina y posteriormente en los Balcanes; la Liga Internacional de la Mujeres por la Libertad y la Paz, el "Sexto Clan" en Somalia, la Ruta Pacífica de las Mujeres en Colombia, entre muchos otros.

${ }^{12}$ El encuentro fue realizado los días 3 y 4 de diciembre de 2012 congregando a 81 organizaciones y redes de mujeres de todo el país. El texto del manifiesto puede ser consultado en https:/www.rutapacifica. org.co/agenda-de-paz/178-la-paz-sin-las-mujeres-no-va-manifiesto-de-mujeres-por-la-paz
} 


\subsection{Repertorio de modalidades de inclusión de las mujeres en el proceso de paz entre el Gobierno Colombiano y las FARC-EP}

Las modalidades de inclusión que predominan en los procesos de paz en el mundo, estudiados por Paffenholz et al. (2016: 6-8) son siete: 1) Representación directa en la mesa de negociaciones, 2) Condición de observadoras, 3) Consultas, 4) Comisiones inclusivas. 5) Talleres de resolución de problemas, 6) Proceso público de toma de decisiones y 7) Acción colectiva. Es importante señalar que pueden presentarse de forma independiente o concomitante en la prenegociación, la negociación y la implementación de los acuerdos de paz.

Las modalidades de inclusión de las mujeres en el proceso de paz entre el Gobierno Colombiano y las FARC-EP están determinadas por una agenda común que les permitió reconocerse y unirse en una voz colectiva que aumentó su capacidad de ejercer influencia en los distintos escenarios. A continuación, se describen cuatro de las siete modalidades de inclusión.

3.1.1. Consultas: esta modalidad de inclusión puede tener lugar en todos los momentos del proceso de paz y puede configurarse una arquitectura de negociación como señala Paffenholz et al. (2016: 31) con distintos formatos de consulta. Así pues puede realizarse a un grupo selecto, a un grupo amplio o pueden ser consultas públicas, si bien arquitectura de negociación del proceso de paz en Colombia incluyó distintos tipos de consultas.

La mesa de conversaciones estableció canales formales de comunicación con la ciudadanía para recibir propuestas en torno a los temas de la agenda de paz a través de formularios físicos y virtuales. De acuerdo a las cifras consolidadas por HUMANAS (2015:40), a partir de los datos suministrados por la Oficina del Alto Comisionado para la Paz (OACP), se evidencia que, de las 5461 propuestas identificadas, solo un $26 \%$ fueron enviadas por mujeres.

Se consultó también a 81 personas expertas (44 hombres y 37 mujeres) en los puntos de la agenda relacionados con 1) Desarrollo agrario integral, 2) Participación política, 3) solución al problema de drogas y 4) víctimas. Las mujeres no fueron consideradas como expertas para los puntos 1 y 2 , de modo que la consideración de experticia estuvo concentrada principalmente en el punto de víctimas, en el que fueron convocadas 36 expertas. Para tratar el punto 4 solo fue considerada una mujer experta (HUMANAS 2015:41).

Por su parte, la subcomisión de género convocó a tres reuniones en la mesa de diálogos de La Habana con representantes de organizaciones de mujeres y LGBTI con trayectorias reconocidas a nivel nacional para conocer, desde su perspectiva, el estado de situación de las mujeres y las personas LGBTI en el marco del conflicto armado siendo solicitadas propuestas y recomendaciones orientadas a fortalecer la perspectiva de género en los acuerdos. 
3.1.2. Comisiones inclusivas: estas comisiones son conformadas comúnmente para la implementación del Acuerdo de Paz, aunque en algunos casos estudiados por Paffenholz et al. (2016: n33) pueden ser constituidas para preparar un proceso de paz o parte de él. El avance de la implementación del Acuerdo Final de Paz en Colombia ha conformado algunas comisiones en las que la inclusión de las mujeres se ha concretado. Nos referiremos a dos de ellas, una, la Instancia Especial para Contribuir a la Implementación del Acuerdo de Paz con un Enfoque de Género; y la otra, la Comisión para el Esclarecimiento de la Verdad, la Convivencia y la No Repetición.

3.1.3.La Instancia Especial: esta comisión para contribuir a la implementación del Acuerdo de Paz con un enfoque de género fue dispuesta en el marco de la creación de la Comisión de Seguimiento, Impulso y Verificación a la Implementación del Acuerdo Final CSIVI ${ }^{13}$ (Decreto 1995, 2016, art. 6).

La conformación de la Instancia especial se hizo a través de una convocatoria nacional a la cual, 830 organizaciones de mujeres postularon ternas territoriales y duplas nacionales. La CSIVI (2017) ${ }^{14}$ constituyó una comisión con el apoyo del mecanismo de acompañamiento internacional al enfoque de género para seleccionar las siete vacantes disponibles para integrar la Instancia. De esta manera, en la Tabla I se indican las mujeres seleccionadas así: cuatro mujeres de organizaciones territoriales, de las cuales, una representa a una organización de víctimas del conflicto armado, dos mujeres de organizaciones de carácter nacional y una de organizaciones LGBTI.

13 Según lo dispuesto por la Mesa de conversaciones, en el punto 6 del Acuerdo Final, la CSIVI está integrada por tres representantes del Gobierno Nacional y tres representantes de las FARC-EP o del partido político que surja de su tránsito a la vida legal. La duración de la Comisión podrá ser hasta de 10 años, acordándose un primer periodo de funcionamiento hasta enero de 2019, fecha a partir de la cual los integrantes de la Comisión decidirán sobre su prórroga. (2017:95)

14 Para ampliar la información sobre el proceso de convocatoria para postular a la instancia especial, puede consultarse en detalle el comunicado conjunto No. 18 de la CSIVI http://www. altocomisionadoparalapaz.gov.co/procesos-y-conversaciones/documentos-y-comunicadosconjuntos/Paginas/Comunicado-Conjunto-No-18.aspx 
Tabla I. Instancia Especial para contribuir a garantizar el enfoque de género en la implementación del Acuerdo Final

\begin{tabular}{|l|l|l|}
\hline Perfil de la organización & Organización & Representante \\
\hline Organizaciones nacionales & Comisión de mujeres indígenas & Victoria Elvira Neuta \\
\cline { 2 - 3 } & Mujeres por la paz & Magda Alberto \\
\hline $\begin{array}{l}\text { Organizaciones de } \\
\text { mujeres a nivel territorial }\end{array}$ & $\begin{array}{l}\text { Unión de ciudadanas colombianas } \\
\text { (Antioquia) }\end{array}$ & Rocío Pineda \\
\cline { 2 - 3 } & $\begin{array}{l}\text { Asociación de Mujeres Araucanas } \\
\text { Trabajadoras. -AmarT- (Arauca) }\end{array}$ & Francisca Aidee Castillo \\
\cline { 2 - 3 } & Mujeres Andinoamazónicas (Putumayo) & Yuly Artunduaga \\
\hline $\begin{array}{l}\text { Organizaciones de mu- } \\
\text { jeres víctimas del conflicto } \\
\text { armado a nivel territorial }\end{array}$ & Narrar para vivir (Bolívar) & Mayerlis Angarita \\
\hline Organizaciones LGBTI & Colombia Diversa & $\begin{array}{l}\text { Marcela Sánchez } \\
\text { Buitrago }\end{array}$ \\
\hline
\end{tabular}

Fuente: Adaptado de CSIVI (2017). [Comunicado conjunto]

La instancia especial se instaló en julio de 2017 y se mantendrá hasta 3 meses adicionales a la finalización de la vigencia del Plan Marco de Implementación del Acuerdo Final y las mujeres que la conformen ejercerán la representación durante dos años ${ }^{15}$ para desarrollar las funciones señaladas a continuación por la CSIVI (2016):

-Dar insumos, sugerir lineamientos y hacer recomendaciones a la CSIVI con el fin de contribuir a garantizar la incorporación del enfoque de género, interseccional y de derechos de las mujeres en la implementación normativa del acuerdo y en el Plan Marco de Implementación, así como en los Planes Cuatrienales de implementación de los acuerdos y en los planes plurianuales de inversión integrados a los planes nacionales y territoriales.

-Hacer seguimiento a la implementación del enfoque de género, interseccional y de derechos de las mujeres de acuerdo con el Plan Marco y los planes cuatrienales de implementación. Este seguimiento deberá ser concretado en informes periódicos y públicos, que serán entregados a la CSIVI y otras instancias.

-Mantener espacios y canales permanentes de diálogo, comunicación e interlocución con las mujeres y las organizaciones, plataformas, colectivos, grupos y redes, cumbres o movimientos a nivel nacional y territorial, para construir propuestas y hacer recomendaciones en relación

15 Solo una tercera parte de las mujeres que integran la Instancia permanecerán al menos un año más en el segundo periodo, con el propósito de propiciar la continuidad en el mecanismo de trabajo. 
a la incorporación del enfoque de género, interseccional y de derechos de las mujeres en la implementación del Acuerdo Final y rendir informes sobre su labor.

-Mantener diálogo constante con la CSIVI, el Consejo Nacional de Reincorporación (CNR), los componentes de verificación y acompañamiento internacional (conformado por ONU Mujeres, la Representante del Secretario General para la Violencia Sexual en el Conflicto, la Federación Democrática Internacional de Mujeres y Suecia), el Instituto Kroc y demás Instancias establecidas por el Acuerdo Final, para garantizar un adecuado seguimiento a la implementación del enfoque de género y de interseccionalidad. Este diálogo se realizará periódicamente y de manera extraordinaria cuando se considere necesario.

\subsubsection{Comisión para el Esclarecimiento de la Verdad, la Convivencia y la No Repetición:}

La creación de esta comisión responde a lo pactado en el punto cinco del Acuerdo Final referido a las víctimas del conflicto armado, el cual pretende lograr la establecer la verdad sobre lo ocurrido, la justicia por los crímenes en el conflicto, la reparación para las víctimas y construir garantías de que no se repitan los hechos.

El comité de escogencia de dicha comisión creado mediante el Decreto 587 de 2017 estableció en su artículo primero:

\footnotetext{
"Los procesos de selección deberán tener en cuenta la participación equitativa entre hombres y mujeres, con respeto de la diversidad étnica y cultural y sujeción a los principios de publicidad, transparencia, participación ciudadana y criterios de cualificación para su selección".
}

Este criterio se aplicó para conformar los mecanismos transicionales del Sistema Integral de Verdad, Justicia, Reparación y No Repetición (SIV-IRNR), dentro de los cuales se encuentra esta Comisión para el Esclarecimiento de la Verdad, la Convivencia y la No Repetición. Siguiendo este criterio y mediante el Decreto 588 de 2017, esta quedó conformada por 11 personas de las cuales 5 son mujeres con amplia trayectoria en la defensa de los derechos humanos y con formación en perspectiva de género, ellas son: Lucía González Duque, Marta Ruiz Naranjo, Ángela Salazar Murillo, María Tobón Yagarí y Alejandra Miller Restrepo.

3.1.4. Proceso público de toma de decisiones: esta modalidad hace referencia a someter el acuerdo de paz logrado en la mesa de negociaciones al voto ciudadano, buscando como señala Paffenholz et al. (2016: 35) "proteger el acuerdo negociado, dotar de legitimidad democrática al proceso y garantizar el apoyo público y la sostenibilidad del acuerdo". 
La convocatoria al plebiscito fue el escenario en el que mujeres de distintos sectores sociales, académicos y políticos, se unieron para impulsar iniciativas ciudadanas a favor de la refrendación de los acuerdos. Una destacada iniciativa fue "un millón de mujeres de paz", en la cual una mujer invitaba a diez mujeres más de su entorno y éstas a su vez convocaban a otras diez y así sucesivamente hasta llegar a la meta del millón de mujeres. Las mujeres se propusieron la refrendación, movilización y construcción de paz mediante actividades de pedagogía, difusión y movilización.

El acuerdo de paz entre Gobierno Colombiano y FARC-EP fue sometido a plebiscito en octubre de 2016 sin que se lograra su refrendación. Los votos por el NO alcanzaron el 50,21\%, superando en 53.784 votos al SI que obtuvo un 49.78\% según datos de la Registraduría Nacional del Estado Civil.

3.1.5. Representación directa en la mesa de negociaciones: en esta modalidad Paffenholz et al. (2016:29) señala que las mujeres pueden formar parte de las delegaciones de las partes principales del conflicto o formar una delegación ellas mismas junto a otras partes. En el caso colombiano, las mujeres participaron como plenipotenciarias en la mesa de diálogo y conformaron una subcomisión de género.

En la fase exploratoria del proceso de paz entre Gobierno Colombiano y FARC-EP, la cual tuvo lugar en Oslo-Noruega en el 2012, Tanja Nijmeijer, de las FARC-EP, integró la delegación. Posteriormente, en el año 2013, en la mesa de diálogos establecida en La Habana, las dos partes incluyen mujeres como delegadas plenipotenciarias. Primero se integra la comandante de las FARC-EP, Victoria Sandino, y posteriormente, María Paulina Riveros y Nigeria Rentería, la primera ejerciendo como directora de Derechos Humanos del Ministerio del Interior y la segunda en calidad de Consejera Presidencial para la Equidad de la Mujer. En la fase final del proceso entra al equipo negociador la Ministra de Relaciones Exteriores María Ángela Holguín.

El ingreso de las mujeres como plenipotenciarias aumentó la posibilidad de incidencia del movimiento social de mujeres sobre la mesa de diálogos y es así como se logró instalar la subcomisión de género, en septiembre de 2014, la cual tuvo como objetivo incorporar la perspectiva de género en los acuerdos a los que se llegara en cada uno de los puntos de la agenda de conversaciones. Para lograr este propósito, la subcomisión de género entre otras acciones, convocó en La Habana a tres reuniones con organizaciones de mujeres y LGBTI y una con expertas nacionales en violencia sexual.

La subcomisión de género definió ocho ejes para la incorporación del enfoque de género en el acuerdo así: 
(1) Acceso y formalización de la propiedad rural en igualdad de condiciones con los hombres.

(2) Garantía de los DESCA de las mujeres y personas con orientación sexual e identidad de género diversa del sector rural.

(3) Promoción de la participación de las mujeres en espacios de representación, toma de decisiones y resolución de conflictos.

(4) Medidas de prevención y protección que atiendan los riesgos específicos de las mujeres.

(5) Acceso a la verdad, a la justicia y a la reparación, y garantías de no repetición.

(6) Reconocimiento público, no estigmatización y difusión de la laboral realizada por las mujeres como sujetas políticas.

(7) Gestión institucional para el fortalecimiento de las organizaciones de mujeres y movimiento LGBTI.

(8) Sistemas de información desagregados. (HUMANAS \& CIASE 2016:34)

Es imporante mencionar que el artículo solo refiere algunas acciones que lograron relevancia nacional e internacional, y no da cuenta de la acción colectiva que las mujeres y sus organizaciones han desplegado en todo el territorio nacional. La suma de estas acciones constituyeron un repertorio que contribuyó a la terminación de un conflicto que si bien arrasó miles de vidas, no les arrebató la esperanza de vivir en un país en paz.

\subsection{Incorporación del enfoque de género en el texto del Acuerdo final}

Las acciones y logros concretos de las mujeres en el proceso de paz en Colombia coinciden con los hallazgos de Paffenholz et al. (2016:25), cuando señalan que la influencia fuerte de las mujeres en los procesos de paz ha contribuido concretamente en cuatro aspectos a saber: 1) cese de hostilidades, inicio o reanudación de negociaciones estancadas, 2) firma de acuerdos de paz ejerciendo presión directa o indirecta en la mesa de negociaciones, 3) mayor representación de las mujeres en el proceso de paz y 4) establecimiento de disposiciones en materia de género.

De este último aspecto da cuenta el Acuerdo final entre el gobierno colombiano y las FARC-EP, en el cual se incorporó el enfoque de género como uno de los principios orientadores para su implementación y se entiende como:

"El reconocimiento de la igualdad de derechos entre hombres y mujeres y de las circunstancias especiales de cada uno, especialmente de las mujeres independientemente de su estado civil, ciclo vital y relación familiar y 
comunitaria, como sujeto de derechos y de especial protección constitucional. Implica en particular la necesidad de garantizar medidas afirmativas para promover esaigualdad, la participación activa de las mujeres y sus organizaciones en la construcción de la paz y el reconocimiento de la victimización de la mujer por causa del conflicto (...).”. La Mesa de Conversaciones (2016:193).

En consecuencia, los seis puntos del Acuerdo disponen entre otras, de las siguientes medidas en materia de género así:

- Punto 1: Hacia un nuevo campo colombiano: propone la promoción de la economía solidaria, la salud sexual y reproductiva y facilitar el acceso a la titularidad de la tierra con prioridad para las mujeres rurales cabeza de familia.

- Punto 2) Participación Política: Apertura democrática para construir la paz, dispone la creación de organizaciones de mujeres, jóvenes y población LGBTI y fomento de la participación de las organizaciones de mujeres, especialmente en instancias de los programas de desarrollo con enfoque territorial y en las circunscripciones especiales transitorias de paz.

- Punto 3) Cese al fuego bilateral y definitivo. Dejación de armas: Señala el compromiso de las partes en no cometer actos de violencia, especialmente aquellos por razón de género, capacitar a todo el personal de monitoreo y verificación en enfoque de género y que dicho personal esté conformado al menos en un $20 \%$ por mujeres.

- $\quad$ Punto 4) Solución al problema de las drogas ilícitas: Pretende incorporar el enfoque diferencial de género a los programas de sustitución de cultivos ilícitos, el diseño de los planes de prevención de drogas y el desarrollo de estrategias como las guarderías infantiles rurales para facilitar el acceso de las mujeres cabeza de familia a oportunidades laborales.

- Punto 5) Víctimas: El sistema integral de verdad, justicia, reparación y no repetición, excluye el indulto para casos de violencia sexual y se regirá por criterios de equidad de género en su composición y disposiciones para promover la participación de las mujeres en la reparación colectiva.

- Punto 6) Implementación, Verificación y refrendación: Se crea una comisión de género, encargada de dar seguimiento y verificar la integración del enfoque transversal de género en la implementación de los acuerdos de paz. 
Es importante señalar que el enfoque de género en el Acuerdo Final no fue incorporado plenamente. como lo impulsaron las organizaciones de mujeres y LGBTI, dado que fue objeto de controversias en la sociedad colombiana, a tal punto, que la oposición al mismo, se convirtió en una de las consignas de la campaña de los promotores del "no" en el plebiscito, por considerarlo una imposición de una "ideología de género" que amenazaba al modelo de familia instituido.

La mayor controversia suscitada en torno a la incorporación del enfoque de género, se dio en relación a las medidas afirmativas para la población LGBTI en el texto del Acuerdo, frente a las cuales, sectores políticos y religiosos solicitaron modificaciones en cuanto a su contenido y alcance. Para citar un ejemplo (OACP 2016:133), argumentos tales como que "la estabilidad social y la legitimidad del acuerdo se evidencia en el apoyo a la protección a la concepción familiar entre un hombre y una mujer", fueron el fundamento para solicitar que se eliminaran del Acuerdo, expresamente, medidas como "la promoción de la no estigmatización en razón de la orientación sexual y la identidad de género diversa".

Es claro, que el camino recorrido para lograr la incorporación del enfoque de género en el Acuerdo final, tuvo avances, retrocesos, inclusiones y exclusiones. En éstas últimas, subyacen estereotipos de género que conducen a un imaginario colectivo de discriminación, que es preciso deconstruir para poder avanzar en la consolidación de una paz estable y duradera.

\section{Transiciones necesarias para la implementación del Acuerdo Final con enfoque de género}

La construcción de la paz en Colombia requiere del impulso y sostenibilidad de transformaciones que cohesionen a una sociedad fragmentada por un conflicto de tan larga duración. La implementación del Acuerdo tiene el potencial para redefinir las relaciones de género y disminuir el riesgo de que las viejas, opresivas y discriminatorias instituciones y prácticas patriarcales sean reestablecidas, en vez de transformadas en el postconflicto (El-Jack, A. 2003: 38).

En este nuevo escenario, la institucionalidad tiene el desafío de operativizar el enfoque de género (ECOSOC, 1997), identificando claramente desde todas las áreas y niveles, las implicaciones diferenciadas que tienen en la vida de los hombres y las mujeres, la legislación, las políticas, los programas y todas las acciones que se emprendan para el mantenimiento de la paz y la reincorporación de excombatientes. La experiencia posbélica de otros países (El-Jack, A. 2003:38), señala que cuando dichas medidas son administradas sin consideración al género pueden, de hecho, exacerbar la inequidad. 
En este mismo sentido, la $\mathrm{R} / 1325$, insta a todos los que participan en los procesos de planificación para el desarme, la desmovilización y la reintegración (DDR) a que tengan presentes las necesidades distintas de las personas excombatientes según sean del género femenino o masculino (ONU, 2000:4). Lo pactado en el Acuerdo Final y posteriormente reglamentado en el Decreto 899 de 2017, coincide con dicha disposición al declarar que el proceso de reincorporación colectiva e individual de las FARC-EP tendrá en todos los componentes un enfoque diferencial con énfasis en los derechos de las mujeres.

Lo anterior resulta fundamental para las mujeres excombatientes dado que por haber ejercido un rol que estereotípicamente se considera masculino y que en consecuencia transgrede los mandatos de género establecidos, son más marginadas que otros grupos de mujeres en sociedades en conflicto y postconflicto (Farr, 2002:34). Las estrategias, instrumentos o iniciativas implementadas en el proceso de reincorporación a la vida civil, también requieren una transición que pueda considerar críticamente las desiguales relaciones y roles de género existentes en una determinada sociedad antes, durante y después del conflicto para evitar reproducir en la sociedad posconflicto las relaciones y roles no igualitarios previamente existentes (Farr, 2002:8).

La reincorporación de los hombres y mujeres excombatientes no puede ser sinónimo de volver a los roles tradicionales de género, sino más bien un instrumento que les brinde posibilidades para redefinir dichos roles en los contextos en los que decidan desarrollar sus proyectos de vida. En ese sentido, el proceso de reintegración requiere también tener una respuesta diferencial no solo a las necesidades del género femenino y masculino, sino también a las de las personas excombatientes con géneros diversos, para que pueden tomar la decisión de redefinir su identidad de género y su orientación sexual, sin la presión de la imposición de las femineidades y las masculinidades militarizadas (CNMH, 2014:111).

La persistencia en el mantenimiento de los roles de género hegemónicos durante el proceso de reincorporación de excombatientes, puede afectar la sostenibilidad de los logros alcanzados en todas las dimensiones del proceso mismo:

"Los hombres ex combatientes, que esperan regresar a retomar su rol de proveedores, se ven confrontados con la realidad de que las mujeres se las arreglan solas (...) las mujeres, luego de haber desempeñado roles no estereotípicos como combatientes, podrían esperar mantener el liderazgo o la independencia que adquirieron durante el conflicto, mientras que los hombres albergan la expectativa de que ellas vuelvan al hogar y continúen en su rol estereotípico de esposa/cuidadora/madre" (El-Jack, A. 2003:35). 
No obstante, la reincorporación con enfoque de género y la transformación de dichos roles no puede centrarse solo en las personas excombatientes, es preciso que en los contextos a donde retornan, también se dirijan acciones para desescalar el impacto que el conflicto armado ha causado en las relaciones comunitarias y la vida cotitidiana. Este enfoque es denominado reintegración comunitaria y al respecto, (Caramés 2008:9) señala que, si los beneficios se centran no únicamente en los excombatientes, sino entre la población que ha sufrido el conflicto, esto contribuirá a la reconciliación y a un mayor y mejor desarrollo de dicha sociedad.

Este enfoque en la reintegración, permite conectar con la dimensión territorial de la construcción de paz, la cual está basada en una nueva alianza entre el Estado y las comunidades para construir conjuntamente institucionalidad en el territorio. La apuesta está fundamentada en la construcción y fortalecimiento de tres niveles de capacidades de los distintos actores en los territorios: 1) capacidades temáticas, 2) capacidades para la gestión territorial y 3) capacidades para la convivencia. Estas últimas fundamentales para la transición, se proponen "superar las estigmatizaciones, la polarización, los rencores y promover las relaciones de confianza, inclusión y equidad. Permitirán un sentir y pensar distintos para lograr un hacer distinto para la paz". (OACP 2017:19)

Las capacidades para la convivencia se desarrollan en cuatro niveles: 1) individual, 2) organizacional, 3) redes y sociedad civil y 4) político social (OACP, 2017: 24). Cuando una sociedad ha estado inserta por más de sesenta años en un conflicto armado, las generaciones presentes no han vivido nunca fuera de éste, lo cual impacta la forma en que se establecen las relaciones cotidianas. En el imaginario colectivo está inserta la desconfianza, el miedo y la prevención hacia las demás personas, impidiendo establecer nuevas formas menos violentas de relacionarse. Por tanto, el primer paso hacia la construcción de paz, se da al gestionar el nivel individual para asumir el desafío de construir en lo cotidiano, relaciones justas y equitativas basadas en la cooperación, la solidaridad y la inclusión.

\section{Conclusiones}

La polémica suscitada con la inclusión del enfoque de género durante el proceso de negociación y firma de los acuerdos entre el Gobierno colombiano y las FARC-EP visibiliza que entre los principales retos para la implementación de éstos, se encuentra la transformación de imaginarios sociales en torno al género, a fin de conseguir que la sociedad colombiana en su conjunto entienda la importancia de integrar mandatos de equidad en la vida cotidiana del país, sentando con ello las bases para conseguir una paz sostenible. 
Colombia cuenta con una gran variedad de disposiciones, normas y políticas en materia de equidad de género, y ha ratificado prácticamente todas las convenciones internacionales al respecto. Sin embargo, si el foco de atención va de lo formal a lo real, no se evidencian impactos o mejoras sustanciales en las condiciones de vida de las mujeres en el país. Se ha mejorado, por ejemplo, en el acceso a la educación o a la salud sexual y reproductiva, no obstante, las cifras de violencia en contra de las mujeres no dejan de aumentar, sigue habiendo desigualdad salarial, un insuficiente acceso al mercado laboral formal y una escasa corresponsabilidad al interior de los hogares. Este reto supone un desafío como sociedad, pues requiere transformar arraigados preceptos culturales que han contribuido a establecer relaciones sociales de forma violenta.

La experiencia previa de los procesos de paz alrededor del mundo, e incluso de todos aquellos intentos tanto exitosos como fallidos con que cuenta la historia de Colombia, son un recordatorio del riesgo permanente de volver atrás en el proceso si se presenta un incumplimiento de lo pactado por las partes. El presente acuerdo entraña uno de los mayores desafíos planteados en la historia de los acuerdos realizados en el país, especialmente en lo referente a la implementación, y dentro de esos desafíos la transversalización del enfoque de género. Experiencias previas han demostrado que, sin las transformaciones necesarias en los diferentes ámbitos sociales, lo pactado pude no alcanzarse poniendo en riesgo la sostenibilidad de la paz.

Las desigualdades sociales y la inequidad de género, contribuyen a perpetuar la violencia, incluso en las etapas de postconflicto, siendo frecuente el aumento de la violencia intrafamiliar y de género en los hogares a los que regresa una persona excombatiente, fruto de los roles y patrones que hacen parte de la jerarquía de las organizaciones armadas. El cambio radical en el uso del tiempo, la búsqueda de estrategias de sustento que mujeres y hombres enfrentan, traen consigo sentimientos de frustración que en muchas ocasiones son aliviados a través de la violencia. Es fundamental, por ende, mirar hacia procesos previos inacabados o fallidos, para aprender de sus límites y contradicciones.

El sinuoso camino recorrido por los grupos de interés a favor de la incorporación del enfoque de género en el proceso de paz estuvo animado por el interés de visibilizar todo aquello que sin la dimensión de género quedaría oculto tras la firma de la paz. El movimiento social de mujeres y las organizaciones LGBTI, lograron visibilizar en la mesa de negociaciones en La Habana, que, en muchos territorios, el repertorio de acciones violentas de los actores armados, se definía también a partir de la identidad de género y de la orientación sexual de las víctimas y, por tanto, los impactos sobre sus vidas eran diferenciados. 
En consecuencia, para contrarrestar esos repertorios violentos y no configurar su identidad exclusivamente en la victimización, se desarrollaron estrategias de resistencia y construcción de paz desde sus territorios. Lograron influenciar la negociación y que el enfoque de género fuera incorporado como principio orientador de la implementación del Acuerdo de Paz. No sin antes atravesar controvertidos debates en los que subyacen imaginarios y prácticas discriminatorias que reflejan las limitaciones que plantea cuestionar los fundamentos culturales sobre los que la sociedad colombiana se ha construido en torno a la comprensión amplia del género.

Esta respuesta social, enciende alertas para que no se detenga la pedagogía para la paz y la insistencia en que la institucionalidad se apropie críticamente de la dimensión de género en esta etapa de postconflicto con las FARC-EP, particularmente, en lo que tiene que ver con la implementación de medidas para la reincorporación de hombres y mujeres excombatientes, quiénes han modificado sus roles de género tras su participación en el conflicto.

Implementar el Acuerdo en las regiones desde una perspectiva de género permitirá avanzar en la disminución de las brechas de desigualdad e inequidad que gestaron y nutrieron el conflicto armado en Colombia. Finalmente, es preciso señalar que si bien se avanzó en materia de inclusión de las mujeres en el proceso de paz y en la disposición de medidas afirmativas que promueven la equidad de género en la implementación del acuerdo, hecho que ha merecido reconocimiento internacional. Al respecto, es necesario decir, que la presencia y participación de las mujeres fue inferior a la de los hombres en gran parte de los espacios de discusión y de toma de decisiones en la mesa de conversaciones. En ese sentido la apuesta por la inclusión debe continuar con más fuerza en la etapa de transición en cada uno de los territorios donde se implementarán los puntos del Acuerdo final para construir un tejido social fuerte, capaz de garantizar la sostenibilidad de la paz.

Por tanto, será necesario realizar transformaciones profundas en áreas fundamentales como la educación para la paz y la convivencia, que permita la disminución de los estereotipos y discriminaciones, la resignificación de los roles tradicionales de género, el respeto a la diversidad en todos los ámbitos, la promoción de la autonomía, empoderamiento y liderazgo de las mujeres, reeducar y sensibilizar a los hombres para el desarrollo de nuevas masculinidades más incluyentes y menos violentas.

A partir de este nuevo escenario en la realidad colombiana, se conjugan en trabajo diario a realizar como ciudadanía, desde la participación activa para la trasformación y el trabajo investigativo que desde el ámbito académico permita la comprensión, tanto de lo sucedido como parte de una memoria histórica necesaria. Ello, para evitar la repetición de un escenario tan cruento en el país y favorecer la identificación de aquellos retos que posibiliten la sostenibilidad 
de la paz y la metamorfosis de la cotidianidad, así como la implementación de proyectos e iniciativas que involucren al conjunto de la sociedad en el proceso de reconstrucción de su tejido social. 


\section{Referencias bibliográficas:}

Arteaga, B. (2012). "El enfoque diferencial: ¡una apuesta para la construcción de paz?" En B. Arteaga, D. Walteros y O. Andrade. (Coords.) Identidades, enfoque diferencial y construcción de paz. Bogotá: Universidad Jorge Tadeo Lozano.

Bonder, G. (1998). Género y Epistemología: Mujeres y Disciplinas. Programa Interdisciplinario de Estudios de Género (PIEG). Chile: Universidad de Chile.

Caramés, A. (2008). Desarme como vínculo entre seguridad y desarrollo La reintegración comunitaria en los programas de Desarme, Desmovilización y Reintegración (DDR) de combatientes en Haití. https://www.ucm.es/ data/cont/docs/430-2013-10-27-PP01-08.pdf

CNMH. (2014). Desafios para la reintegración: enfoques de género, edad y etnia. Bogotá: Imprenta Nacional.

CNMH. (2013). ¡Basta ya! Colombia: memorias de guerra y dignidad. Informe general grupo de memoria histórica. Bogotá: Imprenta Nacional.

Cockburn, C. (2009) [2007] Mujeres ante la guerra. Barcelona: Icaria / Antrazyt.

Consejo de seguridad de Naciones Unidas. (2013). Resolución 2106.

CSIVI. (2017). Comunicado conjunto 18. Instancia Especial para contribuir a garantizar el enfoque de género en la implementación del Acuerdo Final

CSIVI. (2017). Comunicado conjunto. Disponible en: https://www.farc-ep. $\mathrm{co} /$ comunicado/seleccion-de-delegadas-conformacion-de-la-instanciaespecial-de-mujeres.html

Díaz Pérez, A. (2015). Narrativas Hibridas Decoloniales de mujeres colombianas migradas/residentes en Valencia. Tesis doctoral para optar al título de Doctora en Género, Subjetividad, Conocimiento y Cultura. Universidad de Valencia España.

Haraway, D. (1989) Primate Visions: Gender, Race and Nature in the world of Modern Science. Nueva York: Routledge.

Haraway, D. (1991/1995). Ciencia, cyborgs y mujeres. Madrid: Cátedra.

Harding, S. (1986) The Science Question in Feminism. Nueva York: Cornell University Press.

HUMANAS \& CIASE. (2016). Vivencias, aportes y reconocimiento de las mujeres en el proceso de paz en La Habana. Bogotá.

ECOSOC. (1997). Resoluciones y decisiones del Consejo Económico y Social. Nueva York: ONU.

El-Jack, A. (2003). Gender and armed conflict. Overview report. Brighton: Institute of Development Studies. Brighton: University of Sussex. 
Farr, Vanessa (2002). Gendering demilitarization as a Peacebuilding tool. Bonn: BICC.

HUMANAS. (2016). Cumplimiento del Estado Colombiano con la Resolución 1325. Informe de monitoreo año 2015. Bogotá

Ibarra, M. E. (2007) Acciones colectivas de las mujeres en contra de la guerra y por la paz en Colombia. Sociedad y Economía, 13, 66-86.

Iniciativa ciudadana "Un Millón de Mujeres de Paz" comunicado de prensa 14 de febrero de 2016

Instituto Nacional de Medicina Legal y Ciencias Forenses (2016). Boletín Epidemiológico: Violencia de Género en Colombia. Análisis comparativo de las cifras de los años 2014, 2015 y 2016. Colombia.

Lamas, M. (2000). "Diferencias de sexo, género y diferencia sexual". Cuicuilco, $7(18), 1-24$.

Lamas, M. (2007). "Complejidad y claridad en torno al concepto género". En A. Giglia; C. Garma y A. de Teresa (Comp.). ¿Adónde va la antropología? Iztapalapa: División de Ciencias Sociales y Humanidades de la UAM

LIKADI (2004) La inclusión de la perspectiva de género en las políticas locales de Camp de Morvedre. Ayuntamiento de Sagunto. España

Londoño, L. y Fernanda, Y. (2006). Mujeres no contadas, procesos de desmovilización y retorno a la vida civil de mujeres excombatientes en Colombia, 1990-2003. Medellín: La Carretera Social, Instituto de Estudios Regionales, Universidad de Antioquia.

Mendia, I. (2010). Género, rehabilitación postbélica y construcción de la paz. Bilbao: HEGOA. Bilbao.

Meertens, D. (2000). "El futuro nostálgico: desplazamiento, terror y género". Revista Colombiana de Antropología, 36, 112-135.

Meertens, D. (2012). Forced displacement and gender justice in Colombia: Between disproportional effects of violence and historical injustice. Nueva York: ICTJ/Brookings.

Mesa de Conversaciones (2016). Acuerdo final para la terminación del conflicto y la construcción de una paz estable y duradera. Bogotá.

Murguialday, C. (2000) "Género". Diccionario de Acción Humanitaria y Cooperación al Desarrollo. Bilbao: HEGOA.

Naciones Unidas. (2000) Consejo de Seguridad. Resolución 1325.

OACP (2017). Acción Capaz: Estrategia de capacidades para la paz y la convivencia. Bogotá

OACP (2016). Sistematización opciones y propuestas voceros del NO y lo acordado en nuevo acuerdo. Bogotá.

OACP. (sf) La paz es conmigo. Las mujeres como protagonistas en la construcción de paz. Bogotá. 
ONU MUJERES (2017) 100 medidas que incorporan la perspectiva de género en el acuerdo de paz entre el gobierno de Colombia y las Farc-ep para terminar el conflicto y construir una paz estable y duradera. Colombia.

Pedraza, N. (2005). Género, Desplazamiento y Refugio: Frontera Colombia y Venezuela. Bogotá: UNIFEM.

Paffenholz, T., Ross, D. y Schluchter, T (2016). Haciendo que las mujeres cuenten-no solo contando mujeres: evaluación de la inclusión y la influencia de las mujeres en las negociaciones de paz. Ginebra: IPTI \& ONU MUJERES.

Presidencia de la República. (29 de mayo de 2017) Decreto N. ․ 899. Bogotá, Colombia.

Presidencia de la República. (7 de diciembre de 2016) Decreto N.․ 1995. Bogotá, Colombia.

Presidencia de la República. (5 de abril de 2017) Decreto N.․ 587 Bogotá, Colombia.

Rojas, C.yCaro, E. (2002) “Género, conflictoy pazen Colombia: hacia una agenda de investigación. Programa para la Consolidación de la Paz y la Reconstrucción”. http://www.ceipaz.org/images/contenido/G\%C3\%A9nero, $\% 20$ Conflicto $\% 20$ y\%20Paz $\% 20$ en $\% 20$ Colombia $\% 20-\% 20$ hacia $\% 20$ una $\% 20$ agenda $\% 20$ de\%20investigaci\%C3\%B3n_ESP.pdf

Ruta Pacífica de las Mujeres. (2013) La verdad de las mujeres víctimas del conflicto armado en Colombia (resumen). Bogotá: G2 Editores.

Scott, J. (1990). "El género: una categoría útil para el análisis histórico". En J. Amelang y M. Nash (eds.). Historia y género: las mujeres en la Europa moderna y contemporánea. Valencia: Alfons el Magnanim.

Stolcke, V. (2000) “¿Es el sexo para el género lo que la raza para la etnicidad... y la naturaleza para la sociedad?" Politica y Cultura, 14, 25-60.

Viveros, M. (2016) "La interseccionalidad: una aproximación situada a la dominación". Debate feminista, 52, 1-17. 